Check for updates

Cite this: RSC Adv., 2019, 9, 32010

\title{
Oxadiazole derivatives as bipolar host materials for high-performance blue and green phosphorescent organic light-emitting diodes $\uparrow$
}

\author{
Yanming Wang, (D) *ab Keke Duan, ${ }^{a}$ Guoxiang Li ${ }^{a}$ and Gewen Yu ${ }^{a}$
}

By combining two $n$-type groups, pyridine and oxadiazole, with one $\mathrm{p}$-type carbazole group, two novel bipolar hosts, namely 2-(3-(9H-carbazol-9-yl)-[1,1'-biphenyl]-3-yl)-5-(pyridin-2-yl)-1,3,4-oxadiazole (PyOxd-mCz) and 2-(4'-(9H-carbazol-9-yl)-[1,1'-biphenyl]-3-yl)-5-(pyridin-2-yl)-1,3,4-oxadiazole (PyOxd-pCz) have been developed as hosts for blue and green phosphorescent organic light-emitting diodes (PhOLEDs). The two compounds exhibit similar HOMO levels of $-5.64 \mathrm{eV}$ for PyOxd-mCz and $-5.63 \mathrm{eV}$ for PyOxd-pCz and the same LUMO level of $-2.60 \mathrm{eV}$. With a more twisted configuration due to meta connections, PyOxd-mCz possesses a higher triplet energy level $\left(E_{\mathrm{T}}=2.77 \mathrm{eV}\right)$ and more balanced carrier transport than PyOxd-pCz $\left(E_{T}=2.60 \mathrm{eV}\right)$. PyOxd-mCz hosted devices achieve a peak current efficiency of $39.7 \mathrm{~cd} \mathrm{~A}^{-1}$ and a maximum EQE of $20.8 \%$ with a low turn-on voltage of $3.5 \mathrm{~V}$ for Flrpic and $55.2 \mathrm{~cd} \mathrm{~A}^{-1}$ and $16.4 \%$ for $\operatorname{Ir}(\mathrm{ppy})_{3}$. Apart from the appropriate frontier molecular orbital levels and sufficiently high triplet energy of PyOxd-mCz, the more balanced carrier transport plays a key role for excellent device performance.

Received 6th September 2019

Accepted 1st October 2019

DOI: 10.1039/c9ra07129f

rsc.li/rsc-advances balanced charge transport to broaden the recombination zone. $^{\mathbf{1 0 - 1 7}}$ One of the effective ways to obtain balanced charge fluxes is to utilize bipolar host materials. ${ }^{18-23}$ But most bipolar hosts have much higher hole mobilities than electron transporting mobilities even by one or two orders of magnitude, which leads to unbalanced charge transport and narrow recombination zone in emitting layer and eventually leads to low emission efficiency. And in those cases, the ratio of electron-withdrawing groups (n-type) to electron-donating groups (p-type) was usually $1: 1$. However, there have been a few reports about bipolar hosts which have balanced carrier transportation and high external quantum efficiencies (EQE) by adjusting the ratio of n-type groups to p-type groups from $1: 1$ to $2: 1$ or $3: 1$. Chow group had developed a bipolar host POAPF by adjusting the ratio of $n$ type groups to p-type groups to $3: 1$ and a POAPF-based device doped with 7 wt\% FIrpic exhibited a very low turn-on voltage (2.5 V) and high electroluminescence efficiencies (20.6\% and $36.7 \mathrm{~lm}$ $\left.\mathrm{W}^{-1}\right) .{ }^{24} \mathrm{Li}$ group had synthesized several bipolar hosts (the ratio of n-type/p-type $=2: 1$ ) and blue phosphorescent OLEDs based on them achieved high EQE over $20 \%{ }^{25}$ In this paper, we have increased the proportion of n-type groups by attaching two n-type groups of pyridine and oxadiazole to one p-type carbazole group. In this way, we expect to increase the electron mobilities of the hosts and get more balanced charge transporting and high efficiency OLED. The position relationship of carbazole and n-type group on the phenylene bridge is varied as para- and meta-linking mode to tune the molecular conjugated chain length and thus the optoelectronic parameters of the host materials. The blue and green devices based on them exhibit excellent 
performances. The PyOxd-mCz-hosted blue PhOLEDs exhibited a low turn-on voltage of $3.5 \mathrm{~V}$ and a peak current efficiency $\left(\eta_{\mathrm{c}}\right)$ of $39.7 \mathrm{~cd} \mathrm{~A}^{-1}$, a peak power effiency $\left(\eta_{\mathrm{p}}\right)$ of $25.0 \mathrm{~lm} \mathrm{~W}^{-1}$ and a maximum EQE $\left(\eta_{\text {ext }}\right)$ of $20.8 \%$. The PyOxd-mCz-hosted green PhOLEDs exhibited high efficiencies of $55.2 \mathrm{~cd} \mathrm{~A}^{-1}(29.8 \mathrm{~lm} \mathrm{~W}$ and $16.4 \%)$.

\section{Experimental section}

\section{Instruments and methods}

The ${ }^{1} \mathrm{H}$ NMR and ${ }^{13} \mathrm{C}$ NMR spectra were recorded on Bruker AVANCE $500 \mathrm{MHz}$ spectrophotometer and $126 \mathrm{MHz}$ spectrophotometer. The mass spectra were taken on an HP1100LC/ MSD MS spectrometer. The fluorescence and UV-vis absorption spectra measurements were performed on a PerkinElmer LS55 spectrometer and a PerkinElmer Lambda 35 spectrophotometer, respectively. The phosphorescence spectra were measured on an Edinburgh FLS920 spectrometer at $77 \mathrm{~K}$ in 2MeTHF. Thermogravimetric analyses (TGA) and differential scanning calorimetry (DSC) measurements were carried out using a PerkinElmer thermos gravimeter (Model TGA7) and a Netzsch DSC 201 at a heating rate of $10{ }^{\circ} \mathrm{C} \min ^{-1}$ under a nitrogen atmosphere, respectively. The electrochemical measurements were carried out by using a conventional threeelectrode configuration and an electrochemical workstation (BAS100B, USA) at a scan rate of $100 \mathrm{mV} \mathrm{s}^{-1}$. A glass carbon working electrode, a Pt-wire counter electrode, and a saturated calomel electrode (SCE) reference electrode were used. All the electrochemical measurements were carried out at room temperature on samples dissolved in dichloromethane or $\mathrm{N}, \mathrm{N}$ dimethylformamide (DMF), deoxygenated with argon, and with $0.1 \mathrm{M}\left[n \mathrm{Bu}_{4} \mathrm{~N}\right] \mathrm{PF}_{6}$ as the electrolyte.

The electron density distribution of HOMO/LUMO orbitals were optimized by the density functional theory (DFT) using Beck's three-parameterized Lee-Yang-Parr exchange functional (B3LYP) with 6-31G(d) basis sets. There were no imaginary frequencies for both the optimized structures. All these calculations were performed using Gaussian 03. ${ }^{26}$

\section{Compounds synthesis}

The chemical structures and synthetic routes of compounds PyOxd-mCz and PyOxd-pCz are depicted in Scheme 1. The intermediate 2-(3-bromophenyl)-5-(pyridin-2-yl)-1,3,4-oxadiazole (1) was synthesized according to the literatures. ${ }^{27-29}(3-(9 H-$ Carbazol-9-yl) phenyl)boronic acid (2) and (4-(9H-carbazol-9-yl) phenyl)boronic acid (3) were used as received from commercial sources. Through Suzuki cross-coupling reactions, the target compounds PyOxd-mCz and PyOxd-pCz were prepared at yields of $68 \%$ and $81 \%$, respectively. The two compounds both have good solubility in common organic solvents, such as dichloromethane, tetrahydrofuran and ethyl acetate, so that they could be thoroughly purified by column chromatography and repeated recrystallization to reach a high purity for OLED applications. Their chemical structures were fully characterized by ${ }^{1} \mathrm{H}$ NMR, ${ }^{13} \mathrm{C}$ NMR spectroscopy, mass spectrometry, and elemental analysis.

\section{2-(3'-(9H-Carbazol-9-yl)-[1,1'-biphenyl]-3-yl)-5-(pyridin-2-yl)-} 1,3,4-oxadiazole (PyOxd-mCz)

A mixture of 2-(3-bromophenyl)-5-(pyridin-2-yl)-1,3,4-oxadiazole (1.51 g, $5 \mathrm{mmol})$, (3-(9H-carbazol-9-yl)phenyl)boronic acid $(1.58 \mathrm{~g}, 5.5 \mathrm{mmol})$, toluene $(20 \mathrm{~mL})$, ethanol $(5 \mathrm{~mL})$, aqueous sodium carbonate (2 $\mathrm{M}, 4 \mathrm{~mL})$, and tetrakis(triphenylphosphino)palladium(0) $(17.4 \mathrm{mg}, 0.15 \mathrm{mmol})$ were refluxed at $80{ }^{\circ} \mathrm{C}$ under nitrogen atmosphere overnight. After cooling and filtrating, the filtrate was evaporated under reduced pressure. The residue was purified by column chromatography over silica using petroleum ether/ethyl acetate $(5: 1)$ as eluent to give pure PyOxd-mCz as a white solid (1.6 g, 68\% yield).

${ }^{1} \mathrm{H}$ NMR $\left(500 \mathrm{MHz}, \mathrm{CDCl}_{3}\right) \delta=8.79(\mathrm{~d}, J=7.5 \mathrm{~Hz}, 1 \mathrm{H}), 8.48$ $(\mathrm{s}, 1 \mathrm{H}), 8.30(\mathrm{~d}, J=8.0 \mathrm{~Hz}, 1 \mathrm{H}), 8.22(\mathrm{~d}, J=7.5 \mathrm{~Hz}, 1 \mathrm{H}), 8.15(\mathrm{~d}, J$ $=7.5 \mathrm{~Hz}, 2 \mathrm{H}), 7.87(\mathrm{~m}, 2 \mathrm{H}), 7.81(\mathrm{~d}, J=7.5 \mathrm{~Hz}, 1 \mathrm{H}), 7.77(\mathrm{~d}, J=$ $8.0 \mathrm{~Hz}, 1 \mathrm{H}), 7.71(\mathrm{t}, J=7.8 \mathrm{~Hz}, 1 \mathrm{H}), 7.60(\mathrm{~m}, 2 \mathrm{H}), 7.45(\mathrm{t}, 2 \mathrm{H})$, 7.44-7.40 (m, 2H), $7.29(\mathrm{t}, J=7.8 \mathrm{~Hz}, 2 \mathrm{H})$.

${ }^{13} \mathrm{C}$ NMR (126 MHz, $\left.\mathrm{CDCl}_{3}\right) \delta=165.38,163.95,150.25$, 143.54, 141.76, 141.22, 140.87, 138.46, 137.23, 130.69, 130.48, $129.75,126.60,126.33,126.04,125.88,125.84,125.82,124.39$, 123.46, 123.31, 120.35, 120.06, 109.75 .

TOF-EI-MS (m/z): $464.1644[\mathrm{M}]^{+}$. Anal. calcd for $\mathrm{C}_{31} \mathrm{H}_{20} \mathrm{~N}_{4} \mathrm{O}$ : C, 80.15; H, 4.34; N, 12.06. Found: C, 80.18; H, 4.29; N, 12.10.

\section{2-(4'-(9H-Carbazol-9-yl)-[1,1'-biphenyl]-3-yl)-5-(pyridin-2-yl)- 1,3,4-oxadiazole (PyOxd-pCz)}

This compound was synthesized using 2-(3-bromophenyl)-5(pyridin-2-yl)-1,3,4-oxadiazole and (4-(9H-carbazol-9-yl)phenyl) boronic acid as reagents following the above procedure and obtained white solid ( $81 \%$ yield).

${ }^{1} \mathrm{H}$ NMR $\left(500 \mathrm{MHz}, \mathrm{CDCl}_{3}\right) \delta=8.84(\mathrm{~d}, J=4.5 \mathrm{~Hz}, 1 \mathrm{H}), 8.55$ $(\mathrm{s}, 1 \mathrm{H}), 8.36(\mathrm{~d}, J=7.5 \mathrm{~Hz}, 1 \mathrm{H}), 8.26(\mathrm{~d}, J=8.0 \mathrm{~Hz}, 1 \mathrm{H}), 8.16(\mathrm{~d}, J$ $=8.0 \mathrm{~Hz}, 2 \mathrm{H}), 7.94-7.88(\mathrm{~m}, 4 \mathrm{H}), 7.70-7.66(\mathrm{~m}, 3 \mathrm{H}), 7.51-7.48$ $(\mathrm{m}, 3 \mathrm{H}), 7.44(\mathrm{t}, J=8.0 \mathrm{~Hz}, 2 \mathrm{H}), 7.31(\mathrm{t}, J=8.0 \mathrm{~Hz}, 2 \mathrm{H})$.

${ }^{13} \mathrm{C}$ NMR $\left(126 \mathrm{MHz}, \mathrm{CDCl}_{3}\right) \delta=165.56,163.96,150.26$, $143.56,141.39,140.80,138.82$, 137.62, 137.38, 130.66, 129.78, $128.74,127.47,126.44,126.05,125.92,124.37,123.53,123.41$, $120.37,120.10,109.81$.

TOF-EI-MS (m/z): $464.1648[\mathrm{M}]^{+}$. Anal. calcd for $\mathrm{C}_{31} \mathrm{H}_{20} \mathrm{~N}_{4} \mathrm{O}$ : C, 80.15; H, 4.34; N, 12.06. Found: C, 80.21; H, 4.30; N, 12.12.

The ${ }^{1} \mathrm{H}$ NMR and ${ }^{13} \mathrm{C}$ NMR spectra of the compounds are shown as Fig. S3-S8 in the ESI. $\dagger$

\section{Results and discussion}

\section{Thermal prorerties}

Thermal properties of the two materials were investigated by thermogravimetric analysis (TGA) and differential scanning calorimetry (DSC) and the data are summarized in Table 1 . The two compounds exhibit high thermal decomposition temperatures ( $T_{\mathrm{d}}$, corresponding to a $5 \%$ weight loss) over $380{ }^{\circ} \mathrm{C}$ (Fig. S1 $\dagger$ ). And as shown in Fig. 1, the glass transition temperatures $\left(T_{\mathrm{g}}\right)$ for PyOxd-mCz and PyOxd-pCz are $90{ }^{\circ} \mathrm{C}$ and $98{ }^{\circ} \mathrm{C}$, respectively, which are at least $30{ }^{\circ} \mathrm{C}$ higher than that of traditional host material of $\mathrm{mCP}\left(T_{\mathrm{g}}=60^{\circ} \mathrm{C}\right)$. Such high decomposition temperatures and glass transition temperatures will definitely benefit the performances of the emitting devices. 


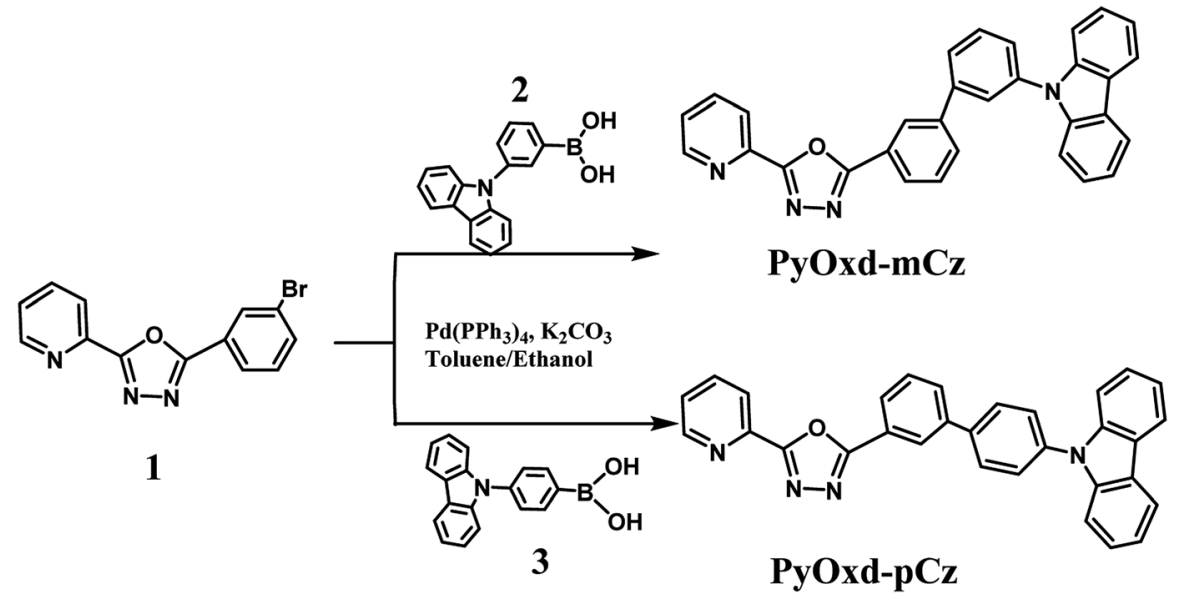

Scheme 1 Chemical structures and synthetic routes of PyOxd-mCz and PyOxd-pCz.

\section{Photophysical properties}

Fig. 2 shows the UV-vis absorption, fluorescence spectra in dilute dichloromethane solutions at $293 \mathrm{~K}$ and the LT PL spectra measured in a frozen 2-methyltetrahydrofuran (2-MeTHF) matrix at $77 \mathrm{~K}$ of PyOxd-mCz and PyOxd-pCz. The UV/ Vis spectra of both PyOxd-mCz and PyOxd-pCz include three main absorption at the wavelength of $243 \mathrm{~nm}, 293 \mathrm{~nm}$ and $340 \mathrm{~nm}$, corresponding to the transitions of phenyl, carbazolecentered $\mathrm{n}-\pi^{*}$ transition and $\pi-\pi^{*}$ transitions of extended $\pi$ conjunction between the carbazole unit and the central phenyl, respectively. ${ }^{30}$ The $340 \mathrm{~nm}$ attribution can be verified by the fact that the absorption of $340 \mathrm{~nm}$ for PyOxd-pCz is stronger than that of PyOxd-mCz, because the former with less space hinder has longer conjunction line which leads to stronger extended $\pi-$ $\pi *$ transition and absorption.

Upon optical excitation at the absorption maxima, PyOxdmCz and PyOxd-pCz emit purple-blue fluorescence with emission peaks at $435 \mathrm{~nm}$ and $441 \mathrm{~nm}$, respectively. The small red shift of 6 nm from PyOxd-mCz to PyOxd-pCz is understandable since the reduced steric hindrance and less twisted molecular backbone for PyOxd-pCz will definitely lead to lower-energy $\mathrm{S}_{1}$ excited state. The low-temperature photoluminescence (LT PL) spectra were measured in the frozen 2-methyltetrahydrofuran glass at $77 \mathrm{~K}$. The triplet energies are estimated from the highest-energy vibronic sub-band of the phosphorescence spectra as ca. $2.77 \mathrm{eV}$ and $2.60 \mathrm{eV}$ for PyOxd-mCz and PyOxd$\mathbf{p C z}$, respectively. It is obvious that the $E_{\mathrm{T}}$ of $\mathbf{P y O x d}-\mathbf{m C z}$ is higher than that of PyOxd-pCz. This can be explained that the less twisted PyOxd-pCz has longer conjugation and thus the lower triplet excited state compared with more steric PyOxdmCz. Their $E_{\mathrm{T}}$ are both high enough for the typical green emitter $\operatorname{Ir}(\mathrm{ppy})_{3}\left(E_{\mathrm{T}}=2.37 \mathrm{eV}\right.$, corresponding to an emission peak at $524 \mathrm{~nm}),{ }^{31}$ but PyOxd-mCz is more appropriate to be the host for traditional blue emitter FIrpic $\left(E_{\mathrm{T}}=2.63 \mathrm{eV}\right.$, corresponding to an emission peak at $472 \mathrm{~nm}){ }^{32,33}$

\section{Electrochemical properties and theoretical calculations}

To investigate the electrochemical properties of the two compounds, cyclic voltammetry (CV) had been performed by using a three-electrode cell setup with $0.1 \mathrm{M} n-\mathrm{Bu}_{4} \mathrm{NPF}_{6}$ as the supporting electrolyte and saturated calomel electrode (SCE) as

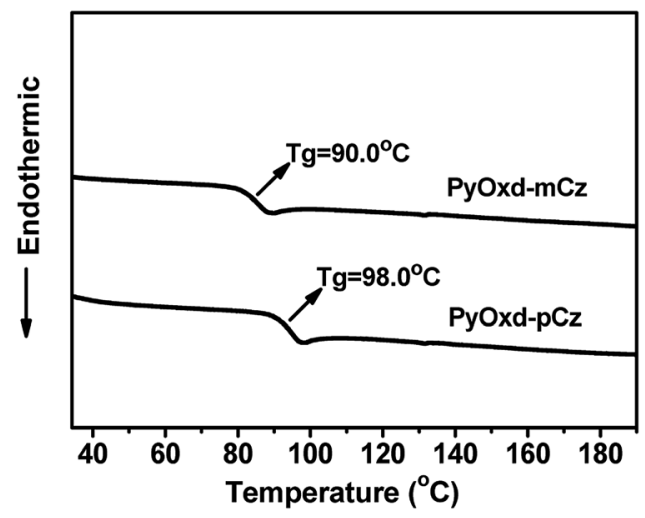

Fig. 1 DSC traces (in the second heating cycle) of PyOxd-mCz and PyOxd-pCz recorded at a heating rate of $10^{\circ} \mathrm{C} \mathrm{min}^{-1}$ under a nitrogen atmosphere.

Table 1 Physical properties of PyOxd-mCz and PyOxd-pCz

\begin{tabular}{llllllll}
\hline Compound & $\operatorname{Abs} \lambda_{\max }{ }^{a}(\mathrm{~nm})$ & $\mathrm{PL} \lambda_{\max }{ }^{a}(\mathrm{~nm})$ & $E_{\mathrm{T}}{ }^{b}(\mathrm{eV})$ & $E_{\mathrm{g}}{ }^{c}(\mathrm{eV})$ & LUMO $(\mathrm{eV})$ & HOMO $(\mathrm{eV})$ & $T_{\mathrm{d}}{ }^{d}\left({ }^{\circ} \mathrm{C}\right)$ \\
\hline PyOxd-mCz & $243,293,340$ & 435 & 2.77 & 3.04 & -5.64 & -2.60 & 395 \\
PyOxd-pCz & $243,293,340$ & 441 & 2.60 & 3.03 & -5.63 & -2.60 & 90 \\
\hline
\end{tabular}

${ }^{a}$ Absorption and fluorescence wavelengths in dilute dichloromethane solutions. ${ }^{b}$ Measured in 2 -Me-THF at $77 \mathrm{~K} .{ }^{c}$ Determined using electrochemical potentials. ${ }^{d} T_{\mathrm{d}}$ is the thermal decomposition temperature corresponding to $5 \%$ weight loss. 

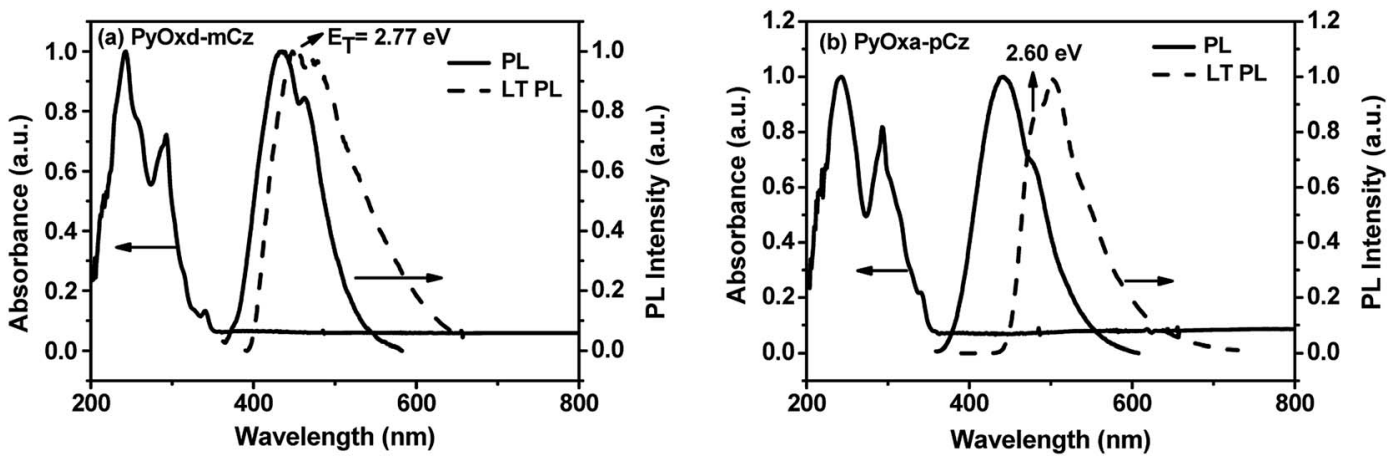

Fig. 2 UV-vis absorption, room-temperature photoluminescence $(\mathrm{PL})$ spectra in dilute dichloromethane solutions, and LT PL spectra in frozen 2-methyltetrahydrofuran matrix at $77 \mathrm{~K}$ of PyOxd-mCz (a) and $\mathrm{PyOxd-pCz} \mathrm{(b).}$

the internal standard. As shown in Fig. 3, PyOxd-mCz and PyOxdpCz both have distinct oxidation and reduction behaviors, which should arise from the electron-donating carbazole unit and electron-withdrawing pyridine-oxadiazole unit, respectively.

The HOMO energies are determined from the onset potential of the first oxidation wave ( $\left.E_{\mathrm{ox}}^{\text {onset }}\right)$ according to the equation of $E_{\mathrm{HOMO}}=-e\left(E_{\mathrm{ox}}^{\mathrm{onset}}+4.4\right)$ as $-5.64 \mathrm{eV}$ and $-5.63 \mathrm{eV}$ for PyOxdmCz and PyOxd-pCz, respectively. The HOMO levels of these compounds are close to that of the widely used hole transport material 1,1-bis[(di-4-tolylamino)phenyl]cyclohexane (TAPC, $-5.60 \mathrm{eV}$ ). The LUMO energies are estimated from the half-wave potentials of the reduction curves to be $-2.60 \mathrm{eV}$ for both PyOxd-mCz and PyOxd-pCz. ${ }^{34}$ The LUMO energies are close to the adjacent electron transport layer 1,3,5-tri $(m$-pyrid-3-ylphenyl)benzene ( $\mathrm{TmPyPb},-2.63 \mathrm{eV}$ ) which will lead to less barrier. Comparing with host materials with one n-type group of oxadiazole (LUMO, $-2.30 \mathrm{eV}$ ), ${ }^{7}$ the two compounds have much lower LUMO energy which indicates that the directly linking pyridine unit to oxadiazole effectively pulls down the LUMO energy of the two hosts. The energy gap $\left(E_{\mathrm{g}}\right)$ of PyOxd-mCz and PyOxd-pCz are determined to be $3.04 \mathrm{eV}$ and $3.03 \mathrm{eV}$, respectively, by calculating the energy differences between the LUMO and HOMO.

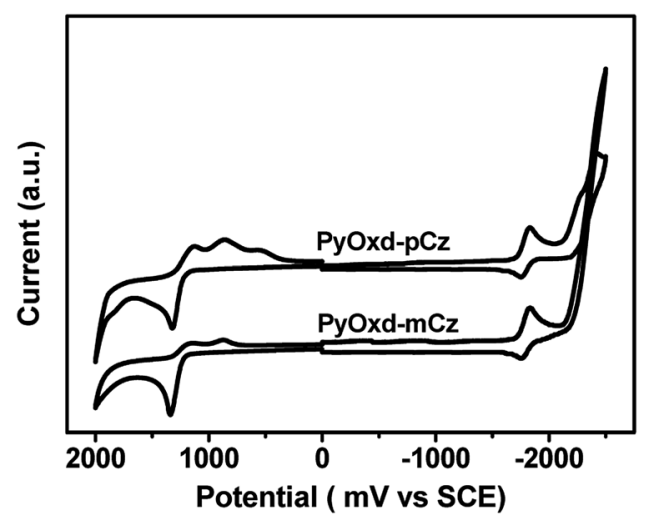

Fig. 3 Cyclic voltammograms of PyOxd-mCz and PyOxd-pCz. The measurement of oxidation potentials were performed in $\mathrm{CH}_{2} \mathrm{Cl}_{2}$ and the reduction $\mathrm{CV}$ were performed in $\mathrm{DMF}$ with $0.1 \mathrm{MnBu}_{4} \mathrm{NPF}_{6}$ as the supporting electrolyte at a scan rate of $100 \mathrm{mV} \mathrm{s}^{-1}$.
The detailed electrochemical and electronic data of the two molecules are listed in Table 1.

B3LYP/6-31G calculations were performed to investigate the influence of the molecular structure on electronic properties. The HOMO/LUMO distributions for the two compounds are given in Fig. 4. Their HOMOs are mainly contributed on the holetransporting carbazole moieties and the adjacent benzene, while the LUMOs are mainly localized on the pyridine and oxadiazole moieties and the adjacent benzene. For PyOxd-mCz, its HOMO and LUMO are completely separated due to larger steric hindrance of meta-substitution; for PyOxd-pCz, there is a tiny overlap on the central phenyl between HOMO and LUMO, which means it has longer $\pi$-conjugation that leads to lower $E_{\mathrm{T}}$. The sufficient spatial separation of the HOMO and LUMO indicates the bipolar charge transporting feature of the two molecules.

\section{Charge-transporting properties}

To evaluate the charge transporting properties of PyOxd-mCz, PyOxd-pCz, the hole-only devices with the structure of ITO/ HATCN $(5 \mathrm{~nm}) /$ TAPC $(5 \mathrm{~nm}) /$ host $(100 \mathrm{~nm}) /$ TAPC $(10 \mathrm{~nm}) / \mathrm{Al}$ $(150 \mathrm{~nm})$ and electron-only devices with the structure of ITO/ TmPyPB $(10 \mathrm{~nm}) /$ host $(100 \mathrm{~nm}) / \mathrm{TmPyPB}(5 \mathrm{~nm}) / \mathrm{LiF}(1 \mathrm{~nm}) / \mathrm{Al}$ $(150 \mathrm{~nm})$ were fabricated, respectively. In hole-only devices, 1,4,5,8,9,11-hexaazatriphenylene hexacarbonitrile (HATCN) was used as a hole-injection layer (HIL), 4,4'-cyclohexylidenebis $[N, N$ bis( $p$-tolyl)aniline] (TAPC) was used as hole-transportation layer (HTL) and TAPC/Al interface was designed to prevent electron injection due to the large electron barrier of $2.3 \mathrm{eV}$. In electrononly devices, a thin layer of TmPyPB was inserted between ITO anode and the host layer to prevent hole injection due to its deep HOMO level $(-6.68 \mathrm{eV})$. The current density versus voltage curves of these single-carrier devices are shown in Fig. 5. Each device exhibited certain current density, implying that both of the compounds possess hole-transporting and electron-transporting abilities, which are the bipolar charge-transporting characters. PyOxd-pCz has much higher electron current densities (109.9 mA $\mathrm{cm}^{-2}$ at $\left.15 \mathrm{~V}\right)$ and hole current densities $\left(3.3 \mathrm{~mA} \mathrm{~cm}{ }^{-2}\right.$ at $\left.30 \mathrm{~V}\right)$ than those of PyOxd-mCz $\left(0.015 \mathrm{~mA} \mathrm{~cm}^{-2}\right.$ at $15 \mathrm{~V}$ for electron and $0.08 \mathrm{~mA} \mathrm{~cm}^{-2}$ for hole at $30 \mathrm{~V}$ ). This can be explained by their different geometrical characteristics. PyOxd-pCz has less twisted configuration and more coplanar conformation, which probably 

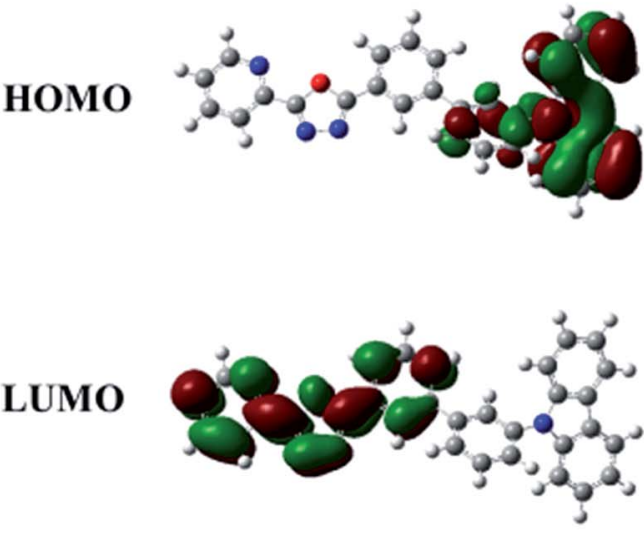

PyOxd-mCz
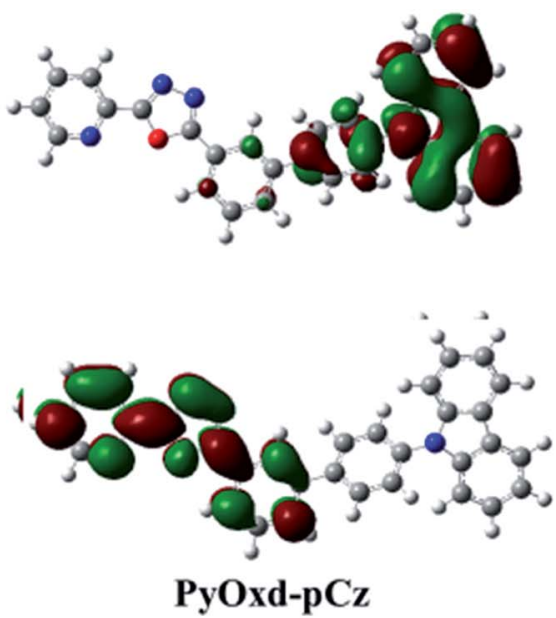

Fig. $4 \mathrm{HOMO}$ and LUMO distributions of PyOxd-mCz and PyOxd-pCz.

leads to intermolecular stacking that are favorable for more rapid charge transportation. But its electron current densities are much higher than hole current densities which will lead to unbalanced charge transportation and inferior device performances. For both PyOxd-mCz and PyOxd-pCz, their electron current densities are higher than hole current densities indicates that attaching an extra electron-efficient pyridine to the host can effectively facilitate the electron transportation.

\section{EL performance of PHOLEDs}

In order to verify the contribution of the double n-type units strategy of the host materials to device performance, we fabricated FIrpic-based blue electrophosphorescent (EL) devices B1 and B2 with the configuration of ITO/HATCN (50 nm)/TAPC (20 $\mathrm{nm}) /$ host: 6 wt\% FIrpic $(20 \mathrm{~nm}) / \mathrm{TmPyPB}(40 \mathrm{~nm}) / \mathrm{LiF}(1 \mathrm{~nm}) / \mathrm{Al}$ $(200 \mathrm{~nm})$. In the two devices, TAPC as the hole-transporting layer (HTL) and TmPyPB as electron-transporting layer (ETL) and hole-blocking layer (HBL), and HATCN and LiF were used as hole- and electron-injecting layers, respectively. Chemical structures of related materials and energy level diagrams of these devices are shown in Fig. S2. $\dagger$ The current densityvoltage-brightness $(J-V-B)$ characteristics and efficiency curves of these devices are shown in Fig. 6. The PyOxd-mCz hosted blue device B1 achieved a better performance with a turn-on voltage $V_{\text {on }}$ (to deliver a brightness of $1 \mathrm{~cd} \mathrm{~m}^{-2}$ ) of $3.5 \mathrm{~V}$, a maximum brightness of $21056 \mathrm{~cd} \mathrm{~m}^{-2}$ at $8.5 \mathrm{~V}$, a maximum current effi-

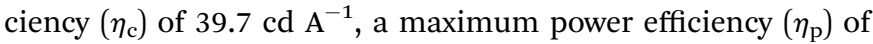
$25.0 \mathrm{~lm} \mathrm{~W}^{-1}$ and a maximum external quantum efficiency $\left(\eta_{\text {ext }}\right)$ of $20.8 \%$ (5 V). The PyOxd-pCz hosted device B2 demonstrated a relatively moderate device performance with a maximum brightness of $15572 \mathrm{~cd} \mathrm{~m}^{-2}(8.5 \mathrm{~V})$, a maximum $\eta_{\mathrm{c}}$ of $28.8 \mathrm{~cd}$ $\mathrm{A}^{-1}$ (corresponding to a peak $\eta_{\text {ext }}$ of $14.6 \%$ and a maximum $\eta_{\mathrm{p}}$ of $20.8 \mathrm{~lm} \mathrm{~W} \mathrm{~W}^{-1}$ at $3.5 \mathrm{~V}$ ). The reason why the PyOxd-mCz-based device has much better performances can be elucidated from the following aspects. First, PyOxd-mCz possesses a high triplet energy $E_{\mathrm{T}}(2.77 \mathrm{eV})$ which is high enough to prevent the adverse back energy transfer from the guest FIrpic $(2.63 \mathrm{eV})$ to the host and efficiently confines the excitons in emissive layer (EML) and guarantees the high efficiencies. Secondly, PyOxd-mCz has more balanced carrier transporting and thus a wide charge recombination zone and high efficiency.

PyOxd-mCz and PyOxd-pCz were also used as hosts to fabricate green phosphorescent devices G1 and G2. The green devices had the same configuration as the above blue ones but with
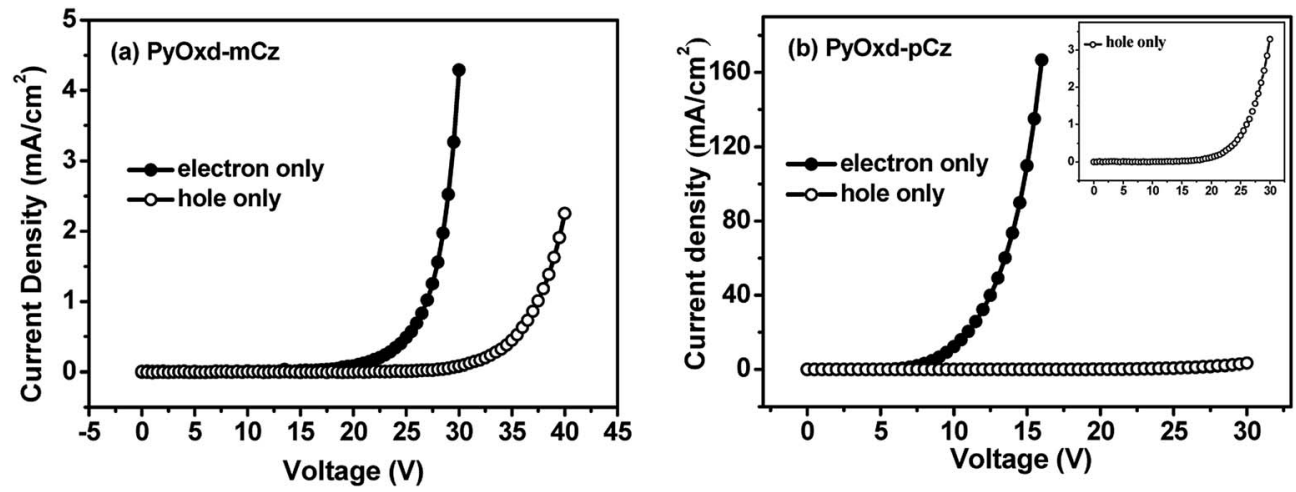

Fig. 5 Current density versus voltage curves of the hole-only and electron-only devices for the compounds PyOxd-mCz (a) and PyOxd-pCz (b). 

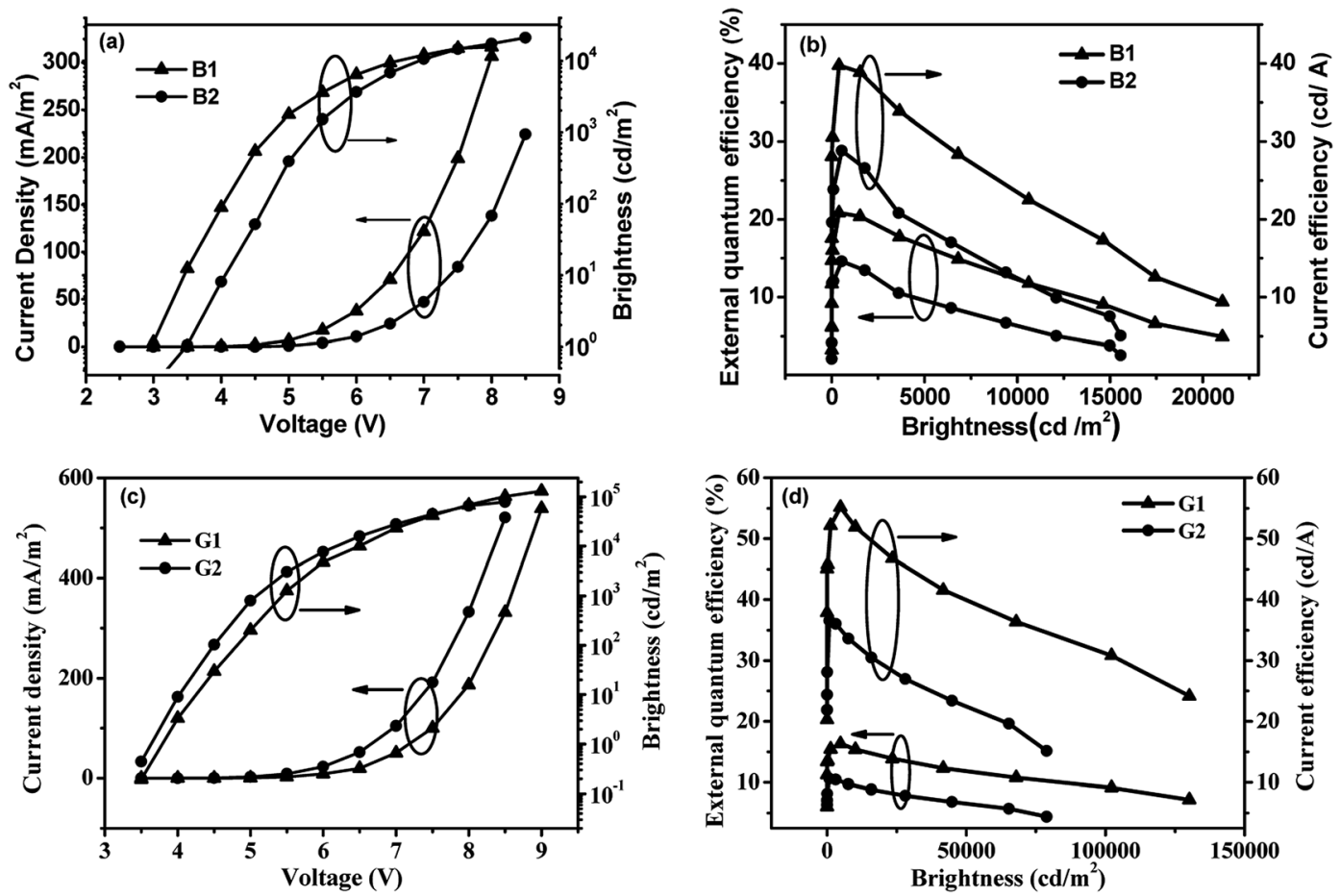

Fig. 6 Current density-voltage-brightness characteristics and efficiency curves for Flrpic-based blue PhOLEDs B1 and B2 (a and b) and for Ir(ppy) ${ }_{3}$-based green PhOLEDs G1 and G2 (c and d).

10 wt $\% \operatorname{Ir}(\text { ppy })_{3}$ instead of FIrpic doped in the hosts as the emitting layers. The J-V-L characteristics and efficiency curves were shown in Fig. 6. Both PyOxd-mCz and PyOxd-pCz hosted devices G1 and G2 showed moderate performances, with a maximum brightness of $130180 \mathrm{~cd} \mathrm{~m}^{-2}$ at $9 \mathrm{~V}$, a maximum current efficiency $\left(\eta_{\mathrm{c}}\right)$ of $55.2 \mathrm{~cd} \mathrm{~A}^{-1}(6 \mathrm{~V})$, a maximum power efficiency $\left(\eta_{\mathrm{p}}\right)$ of $29.8 \mathrm{~lm} \mathrm{~W}^{-1}(5.5 \mathrm{~V})$, and a maximum external quantum efficiency ( $\eta_{\text {ext }}$ ) of $16.4 \%$ ( $6 \mathrm{~V}$ ) for device G1, and 78909 $\mathrm{cd} \mathrm{m}^{-2}, 36.5 \mathrm{~cd} \mathrm{~A}^{-1}, 23.0 \mathrm{~lm}^{-1}$, and 10.6\% for device $\mathrm{G} 2$, respectively. Similar to the case in blue devices, PyOxd-mCz based device has better performances than those of PyOxd-pCz based device. PyOxd-pCz also has enough high $E_{\mathrm{T}}(2.60 \mathrm{eV})$ which can effectively prevent reverse energy transfer from dopant $\operatorname{Ir}(\mathrm{ppy})_{3}$ $\left(E_{\mathrm{T}}=2.37 \mathrm{eV}\right)$ to host as PyOxd-mCz does so the main reason can explain the inferior performances of PyOxd-pCz-hosted green device is the unbalanced electron and hole transportation which leads to narrow recombination zone where triplet-triplet annihilation and exciton-polaron annihilation are easier to take place and eventually leads to lower power efficiency.
The OLEDS performances are summarized in Table 2.

From Table 2, we can see that PyOxd-mCz-hosted devices has lower roll-off than PyOxd-pCz-hosted devices. For example, at a practical brightness of $1000 \mathrm{~cd} \mathrm{~m}^{-2}$, EQE of PyOxd-mCz based device B1 still remains $20.6 \%$, which corresponds to a reduce of 0.9\% from the maximum values. But for PyOxd-mCz based device $\mathrm{B} 2$, EQE is $14.1 \%$ and equals to $3.4 \%$ roll-off at $1000 \mathrm{~cd}$ $\mathrm{m}^{-2}$. There is also an interesting phenomenon as analyzing the performances of the four OLED, that is the blue OLEDs has higher EQE than green OLEDs which is contrary to the previous reports. It can be explained by the two facts. Firstly green emitter $\operatorname{Ir}(\mathrm{ppy})_{3}$ has longer lifetime of $1.9 \mu$ s (ref. 35) than FIrpic $(\tau=1.1 \mu \mathrm{s}),{ }^{36}$ which will lead to higher possibility of triplettriplet annihilation (TTA) and lower efficiency. Secondly there are two requirements for efficient energy transfer from host to guest: one is the overlap between the absorption spectra of guests and the emission spectra of the hosts, which is satisfied for both (see Fig. S9†); the other is the energy overlap between the hosts and the guests. ${ }^{37,38}$ From Fig. S2 $\uparrow$ we can see that

Table 2 Electroluminescence characteristics of blue and green OLEDs

\begin{tabular}{|c|c|c|c|c|c|c|c|c|}
\hline & \multirow[b]{2}{*}{$V_{\text {on }}(\mathrm{V})$} & \multirow[b]{2}{*}{$L_{\max }\left(\mathrm{cd} \mathrm{m}^{-2}\right)$} & \multicolumn{3}{|c|}{ EQE (\%) } & \multicolumn{3}{|c|}{$\eta_{\mathrm{c}}\left(\mathrm{cd} \mathrm{A}^{-1}\right)$} \\
\hline & & & Max. & $1000 \mathrm{~cd} \mathrm{~m}^{-2}$ & $10000 \mathrm{~cd} \mathrm{~m}^{-2}$ & Max. & $1000 \mathrm{~cd} \mathrm{~m}^{-2}$ & $\begin{array}{l}10000 \\
\mathrm{~cd} \mathrm{~m}^{-2}\end{array}$ \\
\hline B1 & 3.5 & 21056 & 20.8 & 20.6 & 12.4 & 39.7 & 39.3 & 23.6 \\
\hline B2 & 3.0 & 15572 & 14.6 & 14.1 & 6.3 & 28.8 & 28.0 & 12.5 \\
\hline G1 & 3.8 & 130180 & 16.4 & 15.1 & 15.4 & 55.2 & 50.5 & 52.0 \\
\hline
\end{tabular}


FIrpic (LUMO/HOMO: $-2.8 \mathrm{eV} /-5.6 \mathrm{eV}$ ) has more energy overlap with the two hosts $(-2.6 \mathrm{eV} /-5.6 \mathrm{eV})$ than $\operatorname{Ir}(\text { ppy })_{3}(-3.0 \mathrm{eV} /$ $-5.4 \mathrm{eV}$ ) does and thus leads to more efficient energy transfer and better device performances.

\section{Conclusion}

Two bipolar host materials, namely PyOxd-mCz and PyOxd-pCz, have been designed and developed for application in blue and green PhOLEDs. Electron transportation can be effectively improved for both of the two compounds by adjusting the ratio of n-type groups to p-type groups from $1: 1$ to $2: 1$. With more twisted configuration by meta-position substitution of carbazole, PyOxd-mCz has higher ET $(2.77 \mathrm{eV})$ and more balanced charge transportation. PyOxd-mCz hosted blue device shows excellent performances with maximum EQE, CE and PE of 20.84\%, 39.7 $\mathrm{cd} \mathrm{A}^{-1}, 25.0 \mathrm{~lm} \mathrm{~W}^{-1}$.

\section{Conflicts of interest}

There are no conflicts to declare.

\section{Acknowledgements}

We acknowledge support from the National Natural Science Foundation of China (21463016) and Science and Technology Research Project of Inner Mongolia Autonomous Region University (NJZY149).

\section{References}

1 S. R. Forrest, Nature, 2004, 428, 911-918.

2 F. May, M. Al-Helwi, B. Baumeier, W. Kowalsky, E. Fuchs, C. Lennartz and D. Andrienko, J. Am. Chem. Soc., 2012, 134, 13818-13822.

3 W. Ji, J. Wang, Q. Zeng, Z. Su and Z. Sun, RSC Adv., 2013, 3, 14616-14621.

4 M. A. Baldo, D. F. O'brien, Y. You, A. Shoustikov, S. Sibley, M. E. Thompson and S. R. Forrest, Nature, 1998, 395, 151.

5 C. Adachi, M. A. Baldo, M. E. Thompson and S. R. Forrest, J. Appl. Phys., 2001, 90, 5048-5051.

6 S. H. Cheng, S. H. Chou, W. Y. Hung, H. W. You, Y. M. Chen, A. Chaskar and K. T. Wong, Org. Electron., 2013, 14, 10861093.

7 M. A. Baldo, C. Adachi and S. R. Forrest, Phys. Rev. B, 2000, 62, 10967-10977.

8 S. Gong, C. Zhong, Q. Fu, D. Ma, J. Qin and C. Yang, J. Phys. Chem. C, 2012, 117, 549-555.

9 S. C. Dong, Y. Liu, Q. Li, L. S. Cui, H. Chen, Z. Q. Jiang and L. S. Liao, J. Mater. Chem. C, 2013, 1, 6575-6584.

10 Y. Tao, Q. Wang, C. Yang, C. Zhong, K. Zhang, J. Qin and D. Ma, Adv. Funct. Mater., 2010, 20, 304-311.

11 S. H. Chou, W. Y. Hung, C. M. Chen, Q. Y. Liu, Y. H. Liu and K. T. Wong, RSC Adv., 2013, 3, 13891-13900.

12 E. Mondal, W. Y. Hung, Y. H. Chen, M. H. Cheng and K. T. Wong, Chem.-Eur. J., 2013, 19, 10563-10572.
13 P. Venkatakrishnan, P. Natarajan, J. N. Moorthy, Z. Lin and T. J. Chow, Tetrahedron, 2012, 68, 7502-7508.

14 H. Huang, Y. Wang, B. Wang, S. Zhuang, B. Pan, X. Yang and C. Yang, J. Mater. Chem. C, 2013, 1, 5899-5908.

15 E. Mondal, W. Y. Hung, H. C. Dai and K. T. Wong, Adv. Funct. Mater., 2013, 23, 3096-3105.

16 R. Braveenth, D. H. Ahn, J. H. Han, J. S. Moon, S. W. Kim, H. Lee and K. Y. Chai, Dyes Pigm., 2018, 157, 377-384.

17 J. Tagare, H. Ulla, M. N. Satyanarayan and S. Vaidyanathan, J. Lumin., 2018, 194, 600-609.

18 Y. T. Lee, Y. T. Chang, M. T. Lee, P. H. Chiang, C. T. Chen and C. T. Chen, J. Mater. Chem. C, 2014, 2, 382-391.

19 Y. Tao, Q. Wang, C. Yang, C. Zhong, K. Zhang, J. Qin and D. Ma, Adv. Funct. Mater., 2010, 20, 304-311.

20 H. Xu, P. Sun, K. Wang, Y. Miao, T. Yang, H. Wang and W. Y. Wong, Tetrahedron, 2016, 72, 4408-4413.

21 K. S. Vadagaonkar, C. J. Yang, W. H. Zeng, J. H. Chen, B. N. Patil, P. Chetti and A. C. Chaskar, Dyes Pigm., 2019, 160, 301-314.

22 L. Xu, Y. Zhao, G. Long, Y. Wang, J. Zhao, D. Li and X. W. Sun, RSC Adv., 2015, 5, 63080-63086.

23 W. Li, J. Li, F. Wang, Z. Gao and S. Zhang, ACS Appl. Mater. Interfaces, 2015, 7, 26206-26216.

24 F. M. Hsu, C. H. Chien, C. F. Shu, C. H. Lai, C. C. Hsieh, K. W. Wang and P. T. Chou, Adv. Funct. Mater., 2009, 19, 2834-2843.

25 W. Li, J. Li, D. Liu and Q. Jin, ACS Appl. Mater. Interfaces, 2016, 8, 22382-22391.

26 M. J. Frisch, G. W. Trucks, H. B. Schlegel, et al., GAUSSIAN 03 (revision C.02), Gaussian, Inc., Wallingford, CT, 2004.

27 S. Megelski, J. S. Stephens, D. B. Chase and J. F. Rabolt, Macromolecules, 2002, 35(22), 8456-8466.

28 H. Tashtoush and R. S. Al-Talib, Magn. Reson. Chem., 1997, 35, 549-552.

29 K. P. Kumar, M. G. Murali and D. Udayakumar, Des. Monomers Polym., 2014, 17, 7-18.

30 C. Fan, F. Zhao, P. Gan, S. Yang, T. Liu, C. Zhong, D. Ma, J. Qin and C. Yang, Chem.-Eur. J., 2012, 18, 5510-5514.

31 W. Li, J. Li, D. Liu, D. Li and F. Wang, ACS Appl. Mater. Interfaces, 2016, 8, 21497-21504.

32 C. Adachi, R. C. Kwong, P. Djurovich, V. Adamovich, M. A. Baldo, M. E. Thompson and S. R. Forrest, Appl. Phys. Lett., 2001, 9, 2082-2084.

33 R. J. Holmes, S. R. Forrest, Y.-J. Tung, R. C. Kwong, J. J. Brown, S. Garon and M. E. Thompson, Appl. Phys. Lett., 2003, 82, 2422-2424.

34 X. K. Liu, C. J. Zheng, M. F. Lo, J. Xiao, Z. Chen, C. L. Liu and X. H. Zhang, Chem. Mater., 2013, 25, 4454-4459.

$35 \mathrm{H}$. Yersin, Highly efficient OLEDs with phosphorescent materials, Wiley-VCH, Weinheim, 2008.

36 M. Sarma, W. L. Tsai, W. K. Lee, Y. Chi, C. C. Wu, P. T. Chou, S. H. Liu and K. T. Wong, Chem, 2017, 3, 461-476.

37 Y. Tao, C. Yang and J. Qin, Chem. Soc. Rev., 2011, 40, 29432970.

38 G. H. Kim, R. Lampande, J. H. Kong, J. M. Lee, J. H. Kwon, J. K. Lee and J. H. Park, RSC Adv., 2015, 5, 31282-31291. 